\section{Vol. 65, No. 52}

In the report "Human Rabies - Puerto Rico, 2015," on page 1476, the first sentence under the Summary heading "What is added by this report?" should have read "A man aged 54 years who was bitten by a mongoose in October 2015 was the first person to acquire rabies from a mongoose in the United States or U.S. territories, confirming mongoose rabies as a public health threat."

\section{Vol. 66, Nos. SS-1 and SS-2}

In the Surveillance Summaries "Leading Causes of Death in Nonmetropolitan and Metropolitan Areas - United States, 1999-2014" and "Reducing Potentially Excess Deaths from the Five Leading Causes of Death in the Rural United States," an error occurred in Figure 5 and Figure 3, respectively. In the last panel of bar charts (stroke), the colors for the left-most set of bars (public health region 1) should be reversed.

In addition, the following person should have been listed as the Guest Editor in the masthead and the Acknowledgments: Robin M. Wagner, PhD, MS, Guest Editor. 